DOI 10.18699/SBB-2020-62

\title{
MGSGenerator 1.5: инструментарий для реконструкции математических моделей метаболических сетей
}

\author{
Казанцев Ф.В. ${ }^{1 *}$, Лашин С.А. ${ }^{1,2}$ \\ ${ }^{1}$ Курчатовский геномный иентр ИЦиГ СО РАН, Новосибирск, Россия \\ ${ }^{2}$ Новосибирский государственный университет, Новосибирск, Россия \\ *e-mail: kazfdr@bionet.nsc.ru
}

Ключевые слова: математические модели, E.coli, API, генерация модели, метаболические сети

\begin{abstract}
Мотивация и цель: Математическое моделирование широко используется в микробиологических исследованиях в задачах анализа метаболических сетей для оценки выхода целевого продукта или подборе оптимальных условий среды. Оно позволяет проверять тысячи вариантов наборов параметров. При моделировании нас интересует не только факт, но и характер взаимодействий: как они протекают в пространстве и во времени. Один из путей получения составных математических моделей - использование фреймовых моделей/стандартных уравнений для серии метаболических процессов. Однако для построения более точных моделей требуется повторно использовать существующие, уже адаптированные к экспериментальным данным модели подсистем. Мы представляем инструмент для получения полной математической модели по выбранной метаболической сети.

Результаты: Для реконструкции фреймовых моделей метаболических сетей мы доработали инструментарий MGSGenerator [1]. Новая версия - 1.5, позволяет выполнять следующие шаги: 1) декомпозиция поданной на вход сети на подсистемы, для последующей обработки; 2) каждая подсистема сравнивается с набором уже существующих моделей подсистем, собранных из нескольких источников. Основной источник готовых моделей - база данных MAMMOTh [2], в которой содержаться ферментативные реакции $E$. coli, адаптированные к экспериментальным данным; 3) если подходящей по структуре моделей не было найдено, запускается механизм построения фреймовых моделей, основанный на серии правил; 4) для того чтобы можно было сразу проводить вычислительные эксперименты, алгоритм добавляет в модель процессы притока в систему и вывода из системы веществ; 5) в конечном итоге получается SBML-модель, которая может быть передана в любой инструмент с поддержкой SBML.

Этот инструментарий был протестирован на серии метаболических сетей разных размеров, от одиночного метаболического пути, до метаболических сетей построенных по данным аннотированного генома бактерий.

Благодарности: Работа выполнена за счет финансирования Курчатовского геномного центра Федерального исследовательского центра ИЦиГ СО РАН, соглашение с Министерством образования и науки РФ № 075-15-2019-1662.
\end{abstract}

\section{Список литературы}

1. Казанцев Ф.В., Акбердин И.Р., Безматерных К.Д., Лихошвай В.А. Система автоматизированной генерации математических моделей генных сетей. Вестник ВОГиС. 2009, 13(1), 163-169.

2. Kazantsev F., I. Akberdin, S. Lashin, N. Ree, V. Timonov, A. Ratushnyi, T. Khlebodarova, Likhoshvai V. MAMMOTh: A new database for curated mathematical models of biomolecular systems. Journal Bioinformatics Computational Biol. 2018, vol. 16(01), pp. 1740010, 2018 https://doi.org/10.1142/S02197200174001. 